\section{Modelling reaction time distribution of fast decision tasks in schizophrenia: Evidence for novel candidate endophenotypes}

Fish, Simon; Toumaian, Maida; Pappa, Eleni; Davies, Timothy J.; Tanti, Ruth; Saville, Christopher; Theleritis, Christos; Economou, Marina; Klein, Christoph; Smyrnis, Nikolaos

\section{Psychiatry Research}

DOI:

10.1016/j.psychres.2018.08.067

Published: 01/11/2018

Peer reviewed version

Cyswllt i'r cyhoeddiad / Link to publication

Dyfyniad o'r fersiwn a gyhoeddwyd / Citation for published version (APA):

Fish, S., Toumaian, M., Pappa, E., Davies, T. J., Tanti, R., Saville, C., Theleritis, C., Economou, M., Klein, C., \& Smyrnis, N. (2018). Modelling reaction time distribution of fast decision tasks in schizophrenia: Evidence for novel candidate endophenotypes. Psychiatry Research, 269, 212220. https://doi.org/10.1016/j.psychres.2018.08.067

\footnotetext{
Hawliau Cyffredinol / General rights

Copyright and moral rights for the publications made accessible in the public portal are retained by the authors and/or other copyright owners and it is a condition of accessing publications that users recognise and abide by the legal requirements associated with these rights.

- Users may download and print one copy of any publication from the public portal for the purpose of private study or research.

- You may not further distribute the material or use it for any profit-making activity or commercial gain

- You may freely distribute the URL identifying the publication in the public portal ?
}

Take down policy

If you believe that this document breaches copyright please contact us providing details, and we will remove access to

the work immediately and investigate your claim. 
Modelling reaction time distribution of fast decision tasks in schizophrenia: evidence for novel candidate endophenotypes

Simon Fish, Maida Toumaian, Eleni Pappa, Timothy J. Davies, Ruth Tanti, Christopher W.N. Saville, Christos Theleritis , Marina Economou, Christoph Klein, Nikolaos Smyrnis

PII:

DOI: S0165-1781(17)32297-7

Reference: https://doi.org/10.1016/j.psychres.2018.08.067 PSY 11661

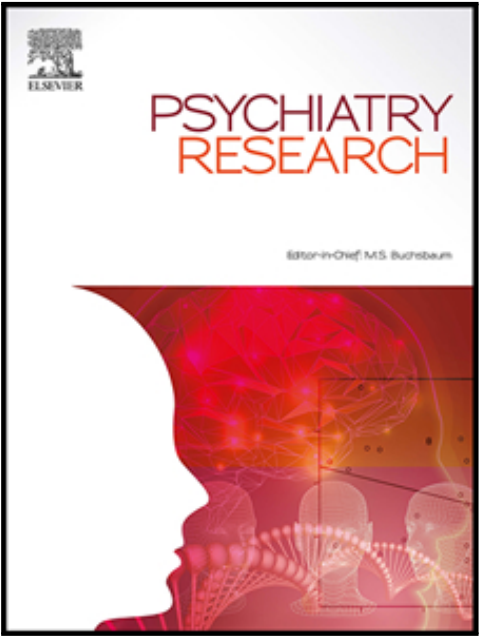

To appear in:

Psychiatry Research

Received date:

13 December 2017

Revised date:

12 August 2018

Accepted date:

16 August 2018

Please cite this article as: Simon Fish, Maida Toumaian, Eleni Pappa, Timothy J. Davies, Ruth Tanti, Christopher W.N. Saville, Christos Theleritis, Marina Economou, Christoph Klein , Nikolaos Smyrnis, Modelling reaction time distribution of fast decision tasks in schizophrenia: evidence for novel candidate endophenotypes, Psychiatry Research (2018), doi: https://doi.org/10.1016/j.psychres.2018.08.067

This is a PDF file of an unedited manuscript that has been accepted for publication. As a service to our customers we are providing this early version of the manuscript. The manuscript will undergo copyediting, typesetting, and review of the resulting proof before it is published in its final form. Please note that during the production process errors may be discovered which could affect the content, and all legal disclaimers that apply to the journal pertain. 
Highlights

- Processing speed measured by Reaction Time (RT) is slower in schizophrenia patients.

- Patients, healthy patient siblings and controls performed two-choice RT tasks.

- Ex-Gaussian RT model and Drift Diffusion Model (DDM) was applied on RT distributions.

- Ex-Gaussian RT parameters dissociated patients and siblings from controls.

- The rising decision signal in DDM dissociated patients and siblings from controls

- RT distribution modeling reveals novel candidate endophenotypes of schizophrenia. 
Modelling reaction time distribution of fast decision tasks in schizophrenia: evidence for novel candidate endophenotypes

Simon Fish ${ }^{\mathrm{a}}$, Maida Toumaian ${ }^{\mathrm{a}}$, Eleni Pappa ${ }^{\mathrm{a}}$, Timothy J. Davies ${ }^{\mathrm{a}}$, Ruth Tanti ${ }^{\mathrm{a}}$, Christopher W.N. Saville $^{\mathrm{c}}$, Christos Theleritis ${ }^{\mathrm{b}}$, Marina Economou ${ }^{\mathrm{b}}$, Christoph Klein ${ }^{\mathrm{d}, \mathrm{e}}$, Nikolaos Smyrnis ${ }^{\mathrm{a}, \mathrm{b}^{*}}$

a. Laboratory of Cognitive Neuroscience, University Mental Health Research Institute, Athens,

Greece.

b. Psychiatry Department, National and Kapodistrian University of Athens, Medical School, Athens, Greece.

c. School of Psychology, Bangor University, United Kingdom.

${ }^{\mathrm{d}}$ Department of Child and Adolescent Psychiatry, Medical Faculty, University of Freiburg, Germany

${ }^{\mathrm{e}}$ Department of Child and Adolescent Psychiatry, Medical Faculty, University of Cologne, Germany

*Nikolaos Smyrnis, Psychiatry Department, National and Kapodistrian University of Athens, Medical School, Eginition Hospital, 72 V. Sofias Ave., Athens, GR-11528, Greece. Tel: +302107289250, Email: smyrnis@med,uoa.gr 


\begin{abstract}
Increased reaction time (RT) and variability of RT in fast decision tasks is observed in patients with schizophrenia and their first degree relatives. This study used modelling of the RT distribution with the aim of identifying novel candidate endophenotypes for schizophrenia. 20 patients with schizophrenia, 15 siblings of patients and 25 healthy controls performed an oddball task of varying working memory load. Increases in mean and standard deviation (SD) of RT were observed for both patients and siblings compared to controls and they were again independent of working memory load. Ex-Gaussian modelling of the RT distribution confirmed that parameters $\mu, \sigma$ and $\tau$ increased significantly in patients and siblings compared to controls. The Drift Diffusion Model was applied on RT distributions. A decrease in the diffusion drift rate $(v)$ modeling the accumulation of evidence for reaching the decision to chose one stimulus over the other, was observed in patients and siblings compared to controls. The mean time of the non-decisional sensorimotor processes $(t 0)$ and it's variance $(s t)$ was also increased in patients and siblings compared to controls. In conclusion modeling of the RT distribution revealed novel potential cognitive endophenotypes in the quest of heritable risk factors for schizophrenia.
\end{abstract}

Key words: Ex-Gaussian model, Drift Diffusion Model, heritable characteristic, working memory, cognitive deficit, psychosis. 


\section{Introduction}

Patients with schizophrenia are slower than healthy controls in a large variety of fast decision tasks in which reaction time (RT) is measured suggesting a core deficit in processing speed for this disorder that is independent of the particular cognitive task being investigated (Nuechterlein, 1977; Cadenhead, 1997). More recently it became evident that the distribution of RT in such fast sensorimotor decision tasks carries more information than can be captured by mean RT alone. One such piece of information of the RT distribution is intra-subject variability (ISV). A particular interest in the study of RT intra-subject variability (RT-ISV) has arisen, proposing that this parameter is a measure of cognitive and sensorimotor processing stability and is not simply a by-product of increased mean RT (Rentrop et al., 2010; Kuntsi and Klein, 2011).

A small number of studies have measured RT-ISV in schizophrenia. In a simple manual response task, mean RT was larger for all groups with psychotic symptoms (schizophrenia and affective disorders), whereas RT-ISV was larger specifically for schizophrenia patients (Schwartz et al., 1989). Vinogradov et al. (1998) showed mean RT and RT-ISV to be dissociated, firstly being a specific predictor of the inability of the patients to maintain a cognitive set, and secondly being a specific predictor of the severity of psychotic and disorganisation symptoms. Kaiser et al. (2008) compared RT-ISV in schizophrenia, major depression and borderline personality disorder patients. The RT-ISV clearly dissociated schizophrenia patients from all other groups. An increase in RT-ISV, but not in mean RT, has also been associated with increased performance errors in patients with schizophrenia and major depression (Van den Bosch et al., 1996; Kaiser et al., 2008). In a visually guided saccade task, increased RT-ISV but not mean RT was observed in patients with schizophrenia compared to healthy controls (Smyrnis et al., 2009). In a later study RT-ISV was modelled as the variation in the decision process leading to the production of a visually guided saccade. An increase in the variation of this decision signal (thus an increase in RT-ISV) but not the increase in mean rise of the signal (corresponding to the mean RT) dissociated patients with schizophrenia from healthy controls, as well as patients with Obsessive Compulsive Disorder (Theleritis et al., 2014). These studies then suggest that increased RT-ISV, like the increase in mean RT, might represent a core 
cognitive deficit in schizophrenia that is independent of the specific cognitive task employed and the motor system used for the response.

One model that more accurately captures the shape of the RT distribution is the ex-Gaussian model (Hohle, 1965; Ratcliff and Murdock, 1976; Luce, 1986; Heathcote et al., 1991). The model is a probability density function formed by the combination of a Gaussian component and an exponential 'tail', providing three basic parameters: $\mathrm{mu}(\mu)$ and sigma $(\sigma)$ correspond to the mean and SD of the Gaussian component, while tau $(\tau)$ signifies the slope of the exponential component. It has been suggested that the $\tau$ component measures the decision element of the underlying cognitive process and the Gaussian components $\mu$ and $\sigma$ measure the non-decisional sensorimotor processes (Hohle, 1965). It seems, however that the relation of the ex-Gaussian parameters to the underlying cognitive processes is more complex (Matzke and Wagenmakers, 2009). Very few studies have used the exGaussian model to investigate RT distribution characteristics in patients with schizophrenia and have had mixed results. In one study $\mu$ and $\tau$ but not $\sigma$ were increased in patients compared $\tau$ o controls (Kieffaber et al., 2006), while in a second study only t was significantly increased in schizophrenia patients compared to controls (Rentrop et al., 2010). In yet another study our group showed a significant increase in $\sigma$ and $\tau$ but not $\mu$ in a simple saccade and manual RT task (Karantinos et al., 2014).

A theoretical model of the underlying cognitive processes that was developed to explain the behaviour both in terms of accuracy and RT distribution characteristics in fast two-choice RT tasks is the Drift Diffusion Model (Ratcliff, 1978; 1979; Ratcliff and McKoon, 2008). The model assumes that RT reflects the time taken for completion of a series of underlying processes (figure 1): a) sensory encoding of the stimulus, b) decision for the selection of the appropriate response between the two possible responses and c) motor planning and execution. The decision process is modelled as the continuous accumulation of information or evidence that "drifts" from a starting point at time zero (no information or no evidence for deciding which stimulus is present) towards a boundary (when enough evidence or information is present for the subject to reach a decision) where the process stops (figure 1). The two possible responses in the task is indicated by the two boundary lines (figure 1) where the information accumulation process stops (upper boundary for responding that the standard stimulus 
was present and lower boundary for responding that the odd stimulus was present). The accumulation of information (drift lines in figure 1) within each trial is noisy and this explains why in some cases the wrong response is selected. The mean rate of the accumulation process, indicated by the slope of the drift line " $v$ " in figure 1, can also vary from trial to trial adding to the total RT variation. The mean drift rate also depends on the stimulus. The accumulation of information is on average slower for more ambiguous stimuli leading to lower mean drift rate as is the case for the odd stimulus in figure 1. The boundary can also change depending on how confident the subject decides to be before terminating the decision process. More confidence is accompanied by larger boundary separation " $a$ " (figure 1) and slower overall decision process. This trade off between boundary separation signifying the level of confidence for a decision to be made and overall decision speed, explains the well-known speed-accuracy trade-off in these tasks. Finally the starting point of the decision process can vary indicating a prior bias towards one of the alternative responses. Overall, the RT distribution is modelled by the mean time for the non-decisional sensory and motor processes and its variance from trial to trial $(t 0, s t O$ in figure 1$)$ and the time for the decision process, which is modelled by the mean drift rate (drift rate $v$ for standard and odd stimulus responses in figure 1), the boundary separation (boundary separation $a$ in figure 1) and the prior bias in favour of one of the two responses at the start of the decision process (starting point " $z$ " in figure 1). Ratcliff and McKoon (2008) provide a comprehensive review of the model.

Over the past decade, the drift diffusion model has been used to model cognitive processes in two choice RT tasks in clinical populations (Ratcliff et al., 2016). The model has been used to demonstrate differences in the rate of the decision process (drift-rate) in individuals with a family history of Alzheimer's disease (Aschenbrenner et al., 2016). A study by White et al. (2010) showed individuals with high anxiety to display larger drift rates for threatening compared to non-threatening words, whereas individuals with low anxiety did not. Research using the model has also been carried out in individuals with ADHD (Mulder et al., 2010; Huang-Pollock et al., 2012; Karalunas et al., 2012; Karalunas and Huang-Pollock, 2013; Metin et al., 2013; Weingard and Huang-Pollock, 2014). The most replicated finding in these studies was a decrease in mean drift rate $(v)$ for patients with ADHD compared to controls. Only one study thus far applied the diffusion drift model of RT in 
schizophrenia patients. Moustafa et al. (2015) applied the model to RT distributions measured in a reward and punishment learning task and reported increased non-decisional processing time $(t O)$, higher separation threshold $(a)$ and a lower drift rate $(v)$ especially for punishment trials in patients compared to controls.

The neurodevelopmental hypothesis of schizophrenia suggests that cognitive deviances observed in patients represent heritable traits that are present before the onset of the disorder and are not the result of its clinical course or the effects of medication (McGrath et al., 2003; Murray and Lewis, 1987; Piper et al., 2012). These deviances have been investigated as potential endophenotypes of the disorder. The endophenotype is a quantitative heritable, trait-related characteristic that is deviant in patients. This characteristic is seen as closer to genetic variation than the symptoms of schizophrenia and thus could be related to heritable risk factors for the disorder (Braff et al 2006). In order to identify potential endophenotypes for schizophrenia, a fundamental criterion is that the deviance observed in patients should also be observed in the unaffected first degree relatives of patients at a higher rate than in the general population (Gottesman and Gould 2003; Braff et al 2006). Earlier studies suggested that higher mean RTs in fast sensorimotor decision tasks can be observed for first degree relatives of patients with schizophrenia (Maier et al., 1994). Much less is known on the heritability of other RT distribution measures in schizophrenia. Hilti et al. (2010) found that siblings of schizophrenia patients had higher RT-ISV than healthy controls on a visual information processing task. To our knowledge the presence of deviances in ex-Gaussian model measures and Drift Diffusion Model measures of RT in first degree relatives of patients with schizophrenia has not been investigated.

The present study aimed to model RT distribution in a simple decision task in schizophrenia patients, their first degree relatives and healthy controls. The hypothesis tested was that RT distribution characteristics and the underlying cognitive processes are similarly deviant in patients and unaffected first degree relatives of patients. The confirmation of this hypothesis would be a crucial first step for identifying these RT distribution deviances as candidate endophenotypes for schizophrenia. Schizophrenia patients, siblings of patients and healthy controls performed a combined 
two-choice oddball, verbal n-back task. Performance accuracy as well as RT distribution parameters was measured for each individual. The RT distributions were then modelled using the ex-Gaussian model as well as the diffusion drift model. RT model parameters were compared among patients, siblings and healthy controls. 


\section{Methods}

\subsection{Participants}

The study sample consisted of 20 patients with schizophrenia (17 men and 3 women), 15 healthy siblings of other untested patients with schizophrenia (4 men and 11 women) and 25 healthy controls (15 men and 10 women). All patients were recruited from the Centre for Rehabilitation of the Psychiatry Department of the National and Kapodistrian University of Athens. Diagnosis was obtained using the Diagnostic Interview for Psychoses (DIP; Castle et al., 2006) with the diagnostic criteria of DSM-IV-TR. All patients but one were treated with antipsychotic medication at the time of testing (mean dose in chlorpromazine equivalents $=590 \mathrm{mg}$, SD: $418 \mathrm{mg}$ ) and were in a stable phase of the disorder. Criteria for exclusion were current treatment with benzodiazepines, additional drug abuse and other organic cerebral illnesses.

First-degree relatives and healthy controls were excluded if they were currently taking any prescribed medication or if they stated any personal history of psychiatric or neurological disorder. A one-way analysis of variance (ANOVA) showed no age difference among groups (patients: Mean=35 years, $\mathrm{SD}=9$; relatives: Mean $=37$ years $\mathrm{SD}=14$; controls: Mean=31 years $\mathrm{SD}=9 ; F(2,57)=1.77$, $p=0.180$ ), as well as no differences in monthly household income, $F(2,39)=1.63, p=0.210$. All participants agreed to take part voluntarily in the study and signed a written informed consent. The study protocol was approved by the ethics committee of the Eginition University Hospital.

\subsection{Stimuli and procedure}

The experiment comprised of two tasks: the 0-back and the 1-back task and two stimulus conditions within each task (oddball: $25 \%$ frequency and standard: $75 \%$ frequency). In the 0-back task the oddball stimulus was the letter ' $\mathrm{O}$ ' and for the 1-back, any stimulus that was identical to the preceding one (Figure 2). Participants responded with their right hand for oddballs and their left hand for standards. Participants were asked to respond as quickly and accurately as possible for all trials. All trials consisted of a letter of the Latin alphabet that appeared every 800 to $1200 \mathrm{~ms}$, for $500 \mathrm{~ms}$. Stimuli were presented in white Courier New typeface, with visual angle of $\sim 3^{\circ}$ on a black 
background, and were delivered using E-Prime 2.0 (Psychology Software Tools, PA, USA). Each participant completed 3 blocks of each task in the sequence A, B, A, B, A, B. Both tasks consisted of 160 trials per block, of which 40 were oddballs and 120 were standards. The order that participants completed the tasks was counterbalanced.

\subsection{Data analysis}

Accuracy was calculated as the percentage of correct responses per task and condition for each subject. RT measured speed of performance only for correct trials. RTs below $120 \mathrm{~ms}$ were removed from the analysis to account for anticipatory responses. Gaussian measures of RT distribution were the Mean RT and RT-SD. These measures were computed for the correct RT distribution for each subject, for each task and condition. The ex-Gaussian model was applied to the correct RT distribution of each subject for each task and stimulus condition and the three parameters of the ex-Gaussian model were derived $(\mu, \sigma$ and $\tau$ ). The model was implemented with the use of the "egfit" function (Lacouture and Cousineau, 2008) in Matlab (Mathworks, version 2014).

We used the freely available "fast-dm-30" program (http://www.psychologie.uniheidelberg.de/ae/meth/fast-dm/; Voss and Voss, 2007; 2008) to compute the parameters of the Drift Diffusion Model of Ratcliff (1978) for each subject. The model was run separately for each block of trials for each task (0-back, 1-back) for each subject. The two stimulus conditions (odd and standard) were used to derive separate drift rates $v$. Thus the program estimated $z r, a, v($ standard $), v(o d d), t 0$ and $s t 0$ for each block and each task as presented in figure 1 . The estimated model values for $v($ standard) were positive while the values for $v($ odd $)$ were negative and they were sign reversed for analysis. The estimated model values for all blocks of each task were then averaged for each subject. We used the Kolmogorov-Smirnov (K-S) test method for estimating the fit of the model. The estimation procedure was successful for all runs of the model ( $p$-values of the K-S test: a) patient group, mean: 0.68 , SD: 0.25 minimum value: 0.065 ; b) relative group, mean: 0.75 , SD: 0.21 , minimum value: 0.151 ; c) control group, mean: 0.70 , SD: 0.22 , minimum value: 0.082 ). 
The means and standard errors of the means for all parameters measured (accuracy, mean RT, RT-SD, ex-Gaussian model and tau and drift diffusion model parameters) for each stimulus condition and each task are presented in supplementary table 1.

A $3 \times 2 \times 2$ mixed-model ANCOVA was run using the General Linear Model for Gaussian measures (mean RT, RT-SD) and ex-Gaussian model parameters $(\mu, \sigma$ and $\tau)$. Group was the independent between-subject variable and task and stimulus condition were the independent withinsubject variables. Since gender differences were present among the different groups (patients, siblings and controls) we used gender as a covariate in the model (introduced as dummy variable 0,1 ). The same ANCOVA model was tested for the drift rate $\mathrm{v}$ of the drift diffusion model. A $3 \times 2$ mixed model ANCOVA was tested for the model parameters $z r, a, t 0$ and $s t 0$. Group was the independent between-subject variable and task was the independent within-subject variable. Again gender was introduced as a covariate.

Planned comparisons were used to test two independent $t$-contrasts for group effects in each ANCOVA analysis. The first contrast combined patients and relatives versus controls and the second contrast tested the difference between patients and relatives while controls were omitted. Based on the hypothesis of this study the first contrast should be significant, indicating that patients and relatives share a common deviance compared to controls. If the second contrast was also significant that would indicate that the presence of the disorder caused a further deviance from controls than that observed in the relatives group.

All statistical analyses were performed using the General Lineal Model module of STATISTICA (v10) statistical software (StatSoft Inc., 1984-2011). 


\section{Results}

Figure 3 presents a characteristic RT-distribution for a single participant from each group (patient, unaffected sibling and control) for each trial type (black bars for standard type trials and grey bars for odd type trials) for each task (right column for 0-back task and left column for 1-back task).

\subsection{Accuracy}

As can be seen in Table 1, all groups responded less accurately on the 1-back compared to the 0 -back task $\left(F_{(1,56)}=41.9, p<10^{-6}, \eta_{\rho}^{2}=0.43\right)$ and for oddball compared to standard trials $\left(F_{(1,56)}=\right.$ $\left.45.0, p<10^{-6}, \eta_{\rho}^{2}=0.44\right)$. Accuracy was also reduced to a greater extent for oddball trials, between the 0-back and 1-back task, compared to standard trials $\left(F_{(1,56)}=17.7, p<10^{-5}, \eta_{\rho}^{2}=0.24\right)$.

Table 1 also shows that accuracy was significantly different among groups $\left(F_{(2,56)}=3.4, p=\right.$ $0.04, \eta_{\rho}^{2}=0.11$ ). Planned comparisons among group means showed that patients when grouped with relatives did not differ from controls while there was a significant decrease of accuracy for patients compared to siblings (Table 2). This difference can be explained by the fact that the decrease in accuracy was only observed in the patient group compared to both the siblings and control groups (table 1).There was no significant group by task interaction $\left(F_{(2,56)}=2.4, p=0.10\right)$, group by stimulus condition interaction $\left(F_{(2,56)}=0.8, p=0.44\right)$, nor a three-way interaction effect $\left(F_{(2,56)}=0.14, p=\right.$ 0.87). Also the effect of gender and the interaction of gender to group and stimulus condition were not significant (results not shown).

\subsection{Gaussian measures of reaction time distribution}

\subsubsection{Mean RT}

Mean RT was significantly larger on the 1-back task compared the 0-back task $\left(F_{(1,56)}=\right.$ 112.7, $\left.p<10^{-6}, \eta_{\rho}^{2}=0.67\right)$ and for oddball compared to standard trials $\left(F_{(1,56)}=21.6, p<10^{-4}, \eta_{\rho}^{2}=\right.$ $0.28)$ (Table 1). There was no significant task by stimulus condition interaction $\left(F_{(1,57)}=0.9, p=\right.$ $0.33)$. 
There was a significant main effect of group for mean $\mathrm{RT}\left(F_{(2,57)}=9.7, p<10^{-3}, \eta_{\rho}^{2}=0.26\right)$. Planned comparisons among group means showed that patients and siblings had a significantly increased mean RT compared to controls while there was no significant difference in mean RT between patients and siblings (Table 2$)$. There was also a significant group by task $\left(F_{(2,56)}=3.6, p=\right.$ $\left.0.03, \eta_{\rho}^{2}=0.26\right)$ while there was no group by stimulus condition interaction $\left(F_{(2,56)}=0.3, p=0.74\right)$ nor a three-way interaction effect $\left(F_{(2,56)} .4, p=0.67\right)$. The group by task interaction was due to the larger increase of mean RT for patients and siblings in the 1-back task versus the 0-back task compared to the increase observed in the control group. By repeating the analysis separately for each task we observed a significant group effect both in the 0-back task $\left(F_{(2,56)}=5.7, p=0.005, \eta_{\rho}^{2}=0.17\right)$ and the 1-back task $\left(F_{(2,56)}=9.7, p=0.002, \eta_{\rho}^{2}=0.15\right)$ with patients and siblings having larger mean RT compared to controls in both tasks. The effect of gender on mean RT and the interaction of gender to task and stimulus condition were not significant (results not shown).

\subsubsection{RT-SD}

The RT-SD was significantly larger for the 1 -back compared to the 0 -back task $\left(F_{(1,56)}=23.2\right.$, $\left.p<10^{-4}, \eta_{\rho}^{2}=0.29\right)$ while the difference between standard and oddball stimuli was not significant $(F$ $(1,56)=3.4, p=0.07)$ (Table 1$)$. There was also a significant task by stimulus condition interaction $\left(F_{(1,}\right.$ 56) $=8.3, p=0.005, \eta_{\rho}^{2}=0.13$ ) and RT-SD was greater for standard compared to oddball trials in the 0-back task while in the 1-back task this difference disappeared.

There was a significant main effect of group for $\operatorname{RT}-\operatorname{SD}\left(F_{(2,57)}=7.9, p<10^{-3}, \eta_{\rho}^{2}=0.22\right)$. Planned comparisons among group means showed that patients and siblings had a significant increase of RT-SD compared to controls while there was no significant difference in RT-SD between patients and siblings (Table 2). There was no significant group by task $\left(F_{(2,56)}=0.4, p=0.65\right)$ group by stimulus condition $\left(F_{(2,56)}=1.5, p=0.23\right)$ or three-way interaction $\left(F_{(2,56)}=0.6, p=0.56\right)$ effect on RT-SD. The effect of gender on mean RT and the interaction of gender to task and stimulus condition were not significant (results not shown). 


\subsection{Ex-Gaussian model parameters}

\subsubsection{Mu $(\mu)$}

Larger $\mu$ values were observed on the 1-back task compared the 0 -back task $\left(F_{(1,56)}=39.0, p\right.$ $\left.<10^{-6}, \eta_{\rho}^{2}=0.41\right)$ and for oddball compared to standard trials $\left(F_{(1,56)}=38.8, p<10^{-6}, \eta_{\rho}^{2}=0.41\right)$ (see Table 1). $\mu$ also increased to a greater extent for oddball trials on the 0 -back task, compared to the 1$\operatorname{back} \operatorname{task}\left(F_{(1,56)}=6.7, p=.012, \eta_{\rho}^{2}=0.10\right)$.

There was a significant main effect of group for $\mu\left(F_{(2,56)}=7.9, p<10^{-3}, \eta_{\rho}^{2}=0.22\right)$. Planned comparisons among group means showed that patients and siblings had a significant increase in $\mu$ compared to controls while there was no significant difference in $\mu$ between patients and siblings (Table 2). There was no significant group by task $\left(F_{(2,56)}=1.5, p=0.23\right)$, group by stimulus condition $\left(F_{(2,56)}=1.2, p=0.30\right)$ nor three-way interaction $\left(F_{(2,56)}=0.5, p=0.60\right)$ effect. The effect of gender on $\mu$ and the interaction of gender to task and stimulus condition were not significant (results not shown).

\subsubsection{Sigma $(\sigma)$}

All groups had larger $\sigma$ scores on the 1-back task compared the 0-back task $\left(F_{(1,56)}=5.7, p\right.$ $\left.=0.020, \eta_{\rho}^{2}=0.09\right)$ and for oddball compared to standard trials $\left(F_{(1,56)}=12.5, p<10^{-3}, \eta_{\rho}^{2}=0.18\right)$. There was no significant task by stimulus condition interaction $\left(F_{(1,56)}=.05, p=0.81\right)$.

There was a significant main effect of group for $\sigma\left(F_{(2,56)}=5.3, p=0.008, \eta_{\rho}^{2}=0.16\right)$. Planned comparisons among group means showed that patients and siblings had a significant increase in $\sigma$ compared to controls while there was no significant difference in $\sigma$ between patients and siblings (Table 2). Finally, there was no significant group by task $\left(F_{(2,56)}=1.7, p=0.19\right)$, group by stimulus condition $\left(F_{(2,56)}=1.6, p=0.21\right)$ or three way interaction $\left(F_{(2,56)}=1.3, p=0.28\right)$ effect. The effect of gender on $\sigma$ and the interaction of gender to task and stimulus condition were not significant (results not shown).

\subsubsection{Tau $(\tau)$}


All groups had larger $\tau$ scores on the 1-back task compared to the 0-back task $\left(F_{(1,56)}=24.8\right.$, $\left.p<10^{-5}, \eta_{\rho}^{2}=0.19\right)$ and for standard compared to oddball trials $\left(F_{(1,56)}=5.8, p=0.030, \eta_{\rho}^{2}=0.08\right)$ (see Table 1). Also $\tau$ was reduced to a greater extent for oddball trials on the 0-back task, compared to the 1-back task $\left(F_{(1,56)}=7.6, \mathrm{p}=0.07, \eta_{\rho}^{2}=0.12\right)$.

There was a significant main effect of group for $\tau\left(F_{(2,56)}=6.4, p=0.003, \eta_{\rho}^{2}=0.19\right)$. Planned comparisons among group means showed that patients and siblings had a significant increase in $\tau$ compared to controls while there was no significant difference in $\tau$ between patients and siblings (Table 2$)$. There was no significant group by task $\left(F_{(2,56)}=0.7, p=0.51\right)$, group by stimulus condition $\left(F_{(2,56)}=2.0, p=0.15\right)$ or three way interaction $\left(F_{(2,56)}=1.0, p=0.38\right)$ effect. The effect of gender on $\tau$ and the interaction of gender to task and stimulus condition were not significant (results not shown).

\subsection{Drift Diffusion Model parameters}

\subsubsection{Starting point bias $(z r)$}

The mean $z r$ values for each task are presented in Table 1. There was a significant effect of task on the starting point bias $\left(F_{1,56}=3293, p<10^{-6}, \eta_{\rho}^{2}=0.37\right)$. There was a small bias towards the odd (matching to previous stimulus) responses in the 1-back task while there was a small bias towards the standard responses in the 0-back task. As can be seen in Table 1 there was no effect on group on the diffusion process starting point bias $z r\left(F_{2,56}=1.6, p=0.20\right)$. There was no significant group by task interaction $\left(F_{2,56}=0.1, p=0.94\right)$. The effect of gender on $z r$ and the interaction of gender to task were not significant (results not shown).

\subsubsection{Boundary Separation (a)}

The threshold separation value $a$, was not different between the two tasks as shown in Table 1 $\left(F_{1,56}=1.2, p=0.27\right)$. The threshold separation $a$, was significantly different among groups $\left(F_{2,56}=\right.$ 3.3, $p=0.044)$. Planned comparisons showed no significant difference for the combined group of patients and siblings versus controls, neither for the difference between patients and siblings (table 2). The effect of the covariate gender on $a$ was significant $\left(F_{1,56}=9.2, p=0.004, \eta_{\rho}^{2}=0.14\right)$ while the 
interaction of gender to task was not significant (results not shown). Women had higher $a$ (mean $=$ 1.27 SE: 0.04$)$ compared to men (mean = 1.14 SE: 0.04).

\subsubsection{Mean drift rate (v)}

Table 1 presents the drift rate $v$ for different stimulus conditions and tasks. The drift rate $v$ was significantly larger for standard compared to oddball trials $\left(F_{1,56}=76.2, p<10^{-6}, \eta_{\rho}^{2}=0.58\right)$ and for 0-back compared to 1-back tasks $\left(F_{1,56}=55.8, p<10^{-6}, \eta_{\rho}^{2}=0.50\right)$. There was also a significant task by group interaction $\left(F_{1,56}=16.9, p<10^{-3}, \eta^{2}{ }_{\rho}=0.23\right)$ due to the fact that the decrease in drift rate for oddball trials was larger in the 1-back task compared to the 0-back task.

As seen in Table 1 there was a significant effect of group on the drift rate $\mathrm{v}\left(F_{2,57}=4.1, p=\right.$ $\left..022, \eta_{\rho}^{2}=0.13\right)$. Planned comparisons among group means showed that patients and siblings had a significantly smaller mean drift rate $v$ compared to controls while there was no significant difference in $v$ between patients and siblings (Table 2). There was no significant group by stimulus condition interaction $\left(F_{1,56}=1.4, p=0.25\right)$ nor group by task interaction $\left(F_{1,56}=1.3, p=0.28\right)$ nor three way interaction $\left(F_{2,56}=1.3, p=0.27\right)$. The effect of gender on $\mathrm{v}$ was not significant $\left(F_{1,56}=0.6, p=0.44\right)$ nor the task by gender interaction $\left(F_{1,56}=0.3, p=0.58\right)$ but there was a significant gender by stimulus condition $\left(F_{1,56}=4.2, p=0.046, \eta_{\rho}{ }_{\rho}=0.07\right)$ and three way interaction (stimulus condition by task by gender $F_{2,56}=4.9, p=0.031, \eta_{\rho}^{2}=0.08$ ). These results were not further explored.

\subsubsection{Non-decisional RT, mean (t0)}

The mean RT for non-decisional processes, $t 0$, was significantly larger for the 1-back compared to the 0 -back task $\left(F_{1,56}=31.6, p<10^{-5}, \eta_{\rho}^{2}=0.36\right)$. There was also a significant effect of group on $t 0\left(F_{2,56}=5.9, p=0.004, \eta_{\rho}^{2}=0.17\right)$. Planned comparisons among group means showed that patients and siblings had a significantly higher $t 0$ compared to controls, and patients had a further significant increase of $\mathrm{t} O$ compared to siblings (Table 2). There was no significant interaction of group and task $\left(F_{2,56}=0.8, p=0.47\right)$. The effect of gender on $t 0$ and the interaction of gender to task were not significant (results not shown). 


\subsubsection{Non-decisional RT, SD (st0)}

There was a significant effect of task on the variability of the non-decisional RT sto $\left(F_{1,56}=\right.$ 13.2, $p<10^{-3}, \eta_{\rho}^{2}=0.19$ ) with larger variability for the 1-back compared to the 0-back task (Table 1). There was also a significant effect of group on $s t O\left(F_{2,56}=9.0, p<10^{-3}, \eta_{\rho}^{2}=0.24\right)$. Planned comparisons among group means showed that patients and siblings had a significant increase in st 0 compared to controls and patients had a significant further increase in $s t O$ compared to siblings (Table 2). There was no significant group by task interaction $\left(F_{2,55}=2.7, p=0.078\right)$. The effect of gender on st 0 and the interaction of gender to task were not significant (results not shown). 


\section{Discussion}

The present study investigated performance in terms of accuracy and speed (RT) in tasks engaging sustained attention (oddball paradigm) and different working memory loads ( 0 and 1 back) of patients with schizophrenia, healthy siblings of patients and a matched group of healthy controls. Our aim was to explore whether specific measures derived from the RT distribution and the underlying cognitive processes differentiate patients as well as siblings from healthy controls suggesting that these measures could be potential cognitive endophenotypes for schizophrenia.

Both mean RT and RT-SD were similarly increased for patients and their unaffected siblings compared to healthy controls. Furthermore both of these effects were not related to stimulus probability. Although there was a significant group by task interaction for mean RT, further analysis showed that the increase of mean RT for patients and siblings compared to controls was present for both the 0-back and 1-back tasks. In a previous study Krieger et al (2005) studied performance of drug naive first episode patients with schizophrenia in a series of visuomotor tasks of increased complexity where the most complex task was the n-back working memory task. They reported only measures of mean RT and showed that mean RT was significantly increased in all tasks in patients compared to controls starting from the simplest visuomotor task. The increasing task complexity led to a further increase of the mean RT difference between patients and controls, similarly to our results concerning mean RT. These results provide evidence that RT distribution differences might represent a general deviance in the speed of cognitive processing in this disorder that is independent of the specific task conditions. This deviance is also present in the unaffected relatives of these patients. Similar increases in mean RT for relatives of patients with schizophrenia have been previously reported (Wang et al., 2007). Hilti et al. (2010) found no difference in mean RT for relatives compared to controls. RT-SD was marginally significantly larger for relatives compared to controls and the inter-quartile RT difference (another measure of RT variability) was significantly larger for the relative group. In that study, however, there was no patient group so the differences in RT distribution measures in the particular task were not compared between patients and relatives. In our study, the increases in mean RT and RT-SD from the control comparison group were similar for both 
schizophrenia patients and a group of siblings of patients, providing evidence that RT distribution measures could be an endophenotype of the disorder.

To our best knowledge, this is the first study employing the ex-Gaussian model of RT distribution to test unaffected first degree relatives of schizophrenia patients. There was an increase in all three measures of the ex-Gaussian model, $\mu$ and $\sigma$ and $\tau$ for both patients and siblings compared to controls. Again these shared deviances in ex-Gaussian model parameters were independent of the stimulus type (standard or odd) and working memory load. In a previous study measuring these parameters in simple visuomotor tasks in patients and controls we observed a significant increase in $\sigma$ and $\tau$ for patients but no increase in $\mu$. In the study of Kieffaber et al. (2006) the authors used an attentional set switching task. They observed increases in both $\mu$ and $\tau$ but not $\sigma$ for patients with schizophrenia compared to controls. In another study Rentrop et al. (2010) observed significant increases only for $\tau$ between patients and controls in a go/no go and continuous performance task. All these previous studies as well as the current study observed an increase of the exponential $(\tau)$ component of the distribution while the deviance regarding the Gaussian normal components $(\mu, \sigma)$ was not replicated in all studies. These findings again suggest that a general deviance in the RT distribution parameters is present in schizophrenia that is independent of the specific task employed. Moreover this is the first study to suggest that this deviance, as revealed with ex-Gaussian modelling of the RT distribution, might be shared with first degree relatives of patients.

The application of the drift diffusion model allowed us to decompose RT into different cognitive processes and study their differences among our groups. We observed a decrease in the mean drift rate for patients independent of task and stimulus condition, suggesting a general deficit in the rate of accumulation of information to reach the decision. Furthermore we showed that this deficit also dissociated patients and siblings from healthy controls. In the only other study that used the Drift Diffusion Model to analyse behaviour in a probabilistic classification learning task in schizophrenia, Moustafa et al (2015) also showed that patients had a significant decrease in mean drift rate $(v)$ that was observed specifically in trials that were followed by punishment. 
The drift rate in the diffusion model can be viewed as a signal that reflects the accumulation of information leading to a decision (Smith and Ratcliff 2004). This signal shares many common characteristics with signals recorded from single neurons in different brain areas of primates during the RT in simple decision tasks (Smith and Ratcliff 2004). A series of studies have used two-choice decision tasks in which primates had to respond by making a saccadic eye movement in the selected target location. Neural firing rate during the RT period in oculomotor areas such as the frontal eye field, the lateral intra-parietal cortical area and the superior colliculus has been viewed as the neural correlate of the behavioural decision process, a neural analogue of the drift diffusion rate (Schall 2003, Gold and Shadlen 2001, Ratcliff et al 2003). In yet another study carried out by Ratcliff et al (2009), it was shown that a specific component of the single trial EEG could be modelled as a neural drift diffusion rate, predicting the behavioral response time and the final choice in a two-choice discrimination task in healthy humans. Speculating on these previous studies one can hypothesize that the neural analogue of a decreased mean drift rate in patients with schizophrenia and their unaffected relatives would be the slower and more variable increase in neuronal activity during the processing of relevant stimulus information in associated areas of the brain. Thus the causes of these differences in drift rate in patients and their unaffected relatives could be investigated by making hypotheses at the neural level. One such hypothesis was tested in the study of Cutsuridis et al (2007). The authors used a biophysical neural network model to simulate the activity of neurons in the antisaccade task. The decision to perform an antisaccade (a saccade in the opposite direction from a presented stimulus) was modeled by a neural signal increasing in time up to a threshold. Variations in the increase of this neural signal predicted RT-distribution characteristics in the performance of this task by healthy adults. More importantly variations in the increase of neuronal activity were 
successfully modeled by variations in specific ionic and synaptic conductances of the modeled cortical neurons.

The increase of the mean non-decision time $(t 0)$ in patients in this study, was also observed in the previous study of Moustafa et al (2015). This increase dissociated patients and siblings from healthy controls but there was also a further increase observed in patients when compared with the unaffected siblings. In the current study an increase in the variance of the non-decisional component of RT ( $s t O$ ) was observed for patients and siblings compared to controls with a further significant increase for patients compared to siblings. In the previous study of Moustafa et al (2015) the variance of the non-decisional time $s t O$ was not included in the model. It is interesting to speculate here that there could be a specific added effect on the non-decisional sensorimotor processing time of the disorder itself. One possible determinant of this added effect could be the influence of antipsychotic medications on the motor system. Thus one could hypothesize that this further increase in the nondecisional component of the RT in the group of medicated patients might not be present in first episode drug naive patients. This hypothesis could be tested in future studies modelling the RT distribution in choice RT tasks in drug naive schizophrenia patients.

We also observed an increase in decision threshold $(a)$ for patients. Planned comparisons of the group means showed that this effect was marginally significant when comparing patients to siblings, thus the difference observed could be the result of increased $a$ only in the patient group. An increase in decision threshold for patients was also observed in the study of Moustafa et al (2015). We also observed that the decision threshold was overall significantly higher in women compared to men, independent of group (patients, relatives or controls).

Although this study focused on RT distribution parameters we will briefly comment on the results of performance accuracy. Patients were less accurate than both controls and relatives independent of task and stimulus condition. Planned comparisons confirmed that patients and siblings did not differ from controls while there was a difference between patients and siblings. These results can be explained by the fact that the decrease in accuracy was observed only in the patient group. Thus the deficit in accuracy of performance in this task for patients with schizophrenia did not 
confirm our initial hypothesis and was not shared with unaffected relatives of patients. Many previous studies have confirmed the deterioration of working memory performance specifically in the n-back task in patients (Manoach, 2003; Carter et al., 1996; Park and Holzman, 1992). Some studies have also confirmed similar performance deficits in relatives of patients (Conklin et al., 2000; Park et al., 1995; Goldberg et al., 2003) while others have failed to do so (Stratta et al., 1997). In line with these latter studies our results showed that performance deficits in patients with schizophrenia in these tasks are only observed in the patient group and are not shared with unaffected relatives. This reduced working memory performance, specifically in the n-back task in patients with schizophrenia, has been linked to dysfunction in prefrontal cortex (Manoach, 2003) with a meta-analysis showing activation differences in the dorsolateral prefrontal cortex, ventrolateral prefrontal cortex, anterior cingulate cortex and left frontal pole (Minzenberg et al., 2009). The fact though that decreased performance accuracy in patients is not shared with relatives suggests that this measure, in contrast to RTdistribution measures cannot be considered as a valid endophenotype candidate.

In conclusion, this study showed that patients with schizophrenia have a deficit in the speed of processing in a simple decision task that is shared with unaffected first degree relatives of patients. Modelling the RT distribution using the ex-Gaussian model confirmed the deviances in patients and relatives. Modelling the cognitive processes underlying the RT distribution using the drift diffusion model, revealed a specific deficit in the rate of accumulation of information for reaching the decision (drift rate) that again was shared in patients and unaffected first degree relatives of patients. These results provide preliminary evidence for the usefulness of modelling the RT distribution to reveal candidate endophenotypes in schizophrenia. They also suggest that a basic deficit in decision processing in this disorder might be heritable. Future studies are needed to confirm these findings and probe the neural and genetic substrate of these deviances. 


\section{References}

Aschenbrenner, A.J., Balota, D.A., Gordon, B.A., Ratcliff, R., Morris, J.C. 2016. A Diffusion Model Analysis of Episodic Recognition in Individuals with a Family History for Alzheimer Disease: The Adult Children Study. Neuropsychology 30(2), 225-238.

Braff, D.L., Freedman, R., Schork, N.J., Gottesman, I.I. Deconstructing Schizophrenia: An Oyerview of the Use of Endophenotypes in Order to Understand a Complex Disorder. Schizophrenia Bulletin $33(1), 21-32$

Cadenhead, K.S., Geyer, M.A., Butler, R.W., Perry, W., Sprock, J., Braff, D L., 1997. Information processing deficits of schizophrenia patients: relationship to clinical ratings, gender and medication status. Schizophrenia Research 28, 51-62.

Carter, C., Robertson, L., Nordahl, T., Chaderjian, M., Kraft, L., O'Shora-Celaya, L., 1996. Spatial working memory deficits and their relationship to negative symptoms in unmedicated schizophrenia patients. Biological Psychiatry 40, 930-932.

Castle, D.J., Jablensky, A., McGrath, J.J., Carr, V., Morgan, V., Waterreus, A. et al., 2006. The diagnostic interview for psychoses (DIP): development, reliability and applications. Psychological Medicine 36, 69-80.

Conklin, H.M., Curtis, C.E., Katsanis, J., Iacono, W.G., 2000. Verbal working memory impairment in schizophrenia patients and their first-degree relatives: evidence from the digit span task. American Journal of Psychiatry 157, 275-277.

Cutsuridis V., Kahramanoglou I., Smyrnis N., Evdokimidis I., Perantonis S., 2007. A biophysical accumulator model of decision making in an antisaccade task. Neurocomputing, 70, 1390-1402. 
Gold, J.I., Shadlen, M.N., 2001. Neural computations that underlie decisions about sensory stimuli. Trends in Cognitive Sciences, 5(1), 10-16.

Goldberg, T.E., Egan, M.F., Gscheidle, T., Coppola, R., Weickert, T., Kolachana, B.S. et al., 2003. Executive subprocesses in working memory: relationship to catechol-O-methyltransferase Val158Met genotype and schizophrenia. Archives of General Psychiatry 60, 889-896.

Gottesman, I.I., Gould, T.D., 2003. The endophenotype concept in psychiatry: etymology and strategic intentions. Am J Psychiatry 160, 636-645.

Heathcote, A., Popiel, S. J., Mewhort, D. J., 1991. Analysis of response time distributions: An example using the Stroop task. Psychological Bulletin 109, (2) 340.

Hilti, C.C., Hilti, L.M., Heinemann, D., Robbins, T., Seifritz, E., Cattapan-Ludewig, K., 2010. Impaired performance on the Rapid Visual Information Processing task (RVIP) could be an endophenotype of schizophrenia. Psychiatry Research 177, 60-64.

Hohle, R. H., 1965. Inferred components of reaction times as functions of foreperiod duration. Journal of experimental psychology 69, (4) 382.

Huang-Pollock, C. L., Karalunas, S. L., Tam, H., Moore, A. N., 2012. Evaluating vigilance deficits in ADHD: a meta-analysis of CPT performance. Journal of Abnormal Psychology 121 (2), 360-371.

Kaiser, S., Roth, A., Rentrop, M., Friederich, H.C., Bender, S., Weisbrod, M., 2008.

Intra-individual reaction time variability in schizophrenia, depression and borderline personality disorder. Brain and Cognition 66, 73-82. 
Karalunas, S. L., Huang-Pollock, C. L., Nigg, J. T., 2012. Decomposing attention-

deficit/hyperactivity disorder (ADHD)-related effects in response speed and variability.

Neuropsychology 26 (6), 684.

Karalunas, S. L., Huang-Pollock, C. L., 2013. Integrating impairments in reaction time and executive function using a diffusion model framework. Journal of abnormal child psychology 41 (5), 837-850.

Karantinos, T., Tsoukas, E., Mantas, A., Kattoulas, E., Stefanis, N., Evdokimidis, T. et al., 2014. Increased intra-subject reaction time variability in the volitional control of movement in schizophrenia. Psychiatry Research 215, 26-32.

Kieffaber, P.D., Kappenman, E.S., Bodkins, M., Shekhar, A., O'Donnell, B.F., Hetrick, W. P., 2006. Switch and maintenance of task set in schizophrenia. Schizophrenia Research 84, 345-358.

Klein, C., Wendling, K., Huettner, P., Ruder, H., Peper, M., 2006. Intra-subject variability in attention-deficit hyperactivity disorder. Biological Psychiatry 60, 1088-1097.

Kofler, M. J., Alderson, R. M., Raiker, J. S., Bolden, J., Sarver, D. E., Rapport, M. D., 2014. Working memory and intraindividual variability as neurocognitive indicators in ADHD: examining competing model predictions. Neuropsychology 28 (3), 459.

Krieger, S., Lis, S., Cetin, T., Gallhofer, B., Meyer-Lindenberg, A. 2005. Executive Function and Cognitive Subprocesses in First-Episode, Drug Naïve Schizophrenia: An Analysis of N-Back Performance. American Journal of Psychiatry, 162, 1206-1208.

Kuntsi, J., Klein, C., 2011. Intra-individual variability in ADHD and its implications for research of causal links. In Stanford, C., Tannock, R. (Eds.), Behavioral 
Neurobiology of ADHD and its Treatment. Current Topics in Behavioral

Neuroscience 9, 67-92.

Lacouture, Y., Cousineau, D., 2008. How to use MATLAB to fit the Ex- Gaussian and other probability functions to a distribution of response times. Tutorials for Quantitative Methods in Psychology 4, 35-45.

Luce, D.R., 1986. Response Times: Their Role in Inferring Elementary Mental Organization, Oxford Psychology Series no. 8. Oxford University Press, New York.

Maier, W., Franke, P., Kopp, B., Hardt, J., Hain, C.H., Rist, F., 1994. Reaction time paradigms in subjects at risk for schizophrenia. Schizophrenia Research 13, 35-43.

Manoach, D.S., 2003. Prefrontal cortex dysfunction during working memory performance in schizophrenia: reconciling discrepant findings. Schizophrenia Research 60, 285-298.

Matzke, D., Wagenmakers, E. J., 2009. Psychological interpretation of the ex-Gaussian and shifted Wald parameters: A diffusion model analysis. Psychonomic bulletin and review 16 (5), 798-817.

McGrath, J. J., Féron, F. P., Burne, T. H., Mackay-Sim, A., Eyles, D. W. 2003. The neurodevelopmental hypothesis of schizophrenia: a review of recent developments. Annals of medicine 35 , (2) 86-93.

Metin, B., Roeyers, H., Wiersema, J. R., van der Meere, J. J., Thompson, M., Sonuga-Barke, E., 2013. ADHD performance reflects inefficient but not impulsive information processing: A diffusion model analysis. Neuropsychology 27 (2), 193. 
Minzenberg, M. J., Laird, A. R., Thelen, S., Carter, C. S., Glahn, D. C. 2009. Meta-analysis of 41 functional neuroimaging studies of executive function in schizophrenia. Archives of general psychiatry 66(8), 811-822.

Moustafa, A. A., Kéri, S., Somlai, Z., Balsdon, T., Frydecka, D., Misiak, B. et al., 2015. Drift diffusion model of reward and punishment learning in schizophrenia: modeling and experimental data. Behavioural brain research 291, 147-154.

Mulder M. J., Bos D., Weusten J.M.H., van Belle J., van Dijk S.C., Simen P. et al., 2010. Basic Impairments in Regulating the Speed-Accuracy

Tradeoff Predict Symptoms of Attention-Deficit/Hyperactivity Disorder. Biological Psychiatry 68, $1114-1119$.

Murray, R. M., Lewis, S. W. 1987. Is schizophrenia a neurodevelopmental disorder? British Medical Journal (Clinical Research Ed.) 295 (6600), 681.

Nuechterlein, K.H., 1977. Reaction time and attention in schizophrenia: a critical evaluation of the data and theories. Schizophrenia Bulletin 3, 373-428.

Park, S., Holzman, P.S., 1992. Schizophrenics show spatial working memory deficits. Archives of General Psychiatry 49, 975-982.

Park, S., Holzman, P.S., Goldman-Rakic, P.S., 1995. Spatial working memory deficits in the relatives of schizophrenic patients. Archives of General Psychiatry 52, 821-828.

Piper, M., Beneyto, M., Burne, T. H., Eyles, D. W., Lewis, D. A., McGrath, J. J. 2012. The neurodevelopmental hypothesis of schizophrenia: convergent clues from epidemiology and neuropathology. Psychiatric Clinics of North America 35 (3), 571-584. 
Ratcliff, R., Murdock, B. B., 1976. Retrieval processes in recognition memory. Psychological Review $83(3), 190$.

Ratcliff, R., 1978. A theory of memory retrieval. Psychological review 85, (2) 59.

Ratcliff, R., 1979. Group reaction time distributions and an analysis of distribution statistics. Psychological Bulletin 86, 446-461.

Ratcliff, R., McKoon, G., 2008. The diffusion decision model: theory and data for two-choice decision tasks. Neural computation 20 (4), 873-922.

Ratcliff, R., Philiastides, Sajda, P., 2009. Quality of evidence for perceptual decision making is indexed by trial-to-trial variability of the EEG, PNAS 106(16), 6539-6544.

Ratcliff, R., Smith, P. L., Brown, S. D., McKoon, G., 2016. Diffusion decision model: current issues and history. Trends in cognitive sciences 20 (4), 260-281.

Rentrop, M., Rodewald, K, Roth, A., Simon, J., Walther, S., Fiedler, P. et al., 2010. Intra-individual variability in high-functioning patients with schizophrenia. Psychiatry Research 178 27-32.

Schall, J.D., 2003. Neural correlates of decision processes: neural and mental chronometry. Current Opinion in Neurobiology 13, 182-186.

Schwartz, F., Carr, A.C., Munich, R.L., Glauber, S., Lesser, B., Murray, J., 1989. Reaction time impairment in schizophrenia and affective illness: the role of attention. Biological psychiatry 25(5), 540-548. 
Smith, P.L., Ratcliff, R., 2004. Psychology and neurobiology of simple decisions. Trends in Neuroscience 27(3), 161-168.

Smyrnis, N., Karantinos, T., Malogiannis, I., Theleritis, C., Mantas, A., Stefanis, N.C. et al., 2009. Larger variability of saccadic reaction times in schizophrenia patients. Psychiatry Research 168, 129136.

Stratta, P., Daneluzzo, E., Mattei, P., Bustini, M., Casacchia, M., Rossi, A., 1997. No deficit in Wisconsin Card Sorting Test performance of schizophrenic patients' first-degree relatives. Schizophrenia Research 26, 147- 151.

Theleritis, C., Evdokimidis, I., Smyrnis, N., 2014. Variability in the decision process leading to saccades: a specific marker for schizophrenia? Psychophysiology 51, 327-336.

Van den Bosch, R.J., Rombouts, R.P.,Van Asma, M.J., 1996. What determines continuous performance task performance? Schizophrenia Bulletin 22, 643-651.

Vinogradov, S., Poole, J.H., Willis-Shore, J., Ober, B.A., Shenaut, G.K., 1998. Slower and more variable reaction times in schizophrenia: what do they signify?

Schizophrenia Research 32, 183-190.

Voss, A., Voss, J., 2007. Fast-dm: A free program for efficient diffusion model analysis. Behavior Research Methods 39 (4), 767-775.

Voss, A., Voss, J., 2008. A fast numerical algorithm for the estimation of diffusion model parameters. Journal of Mathematical Psychology 52 (1), 1-9. 
Wang, Q., Chan, R., Sun, J., Yao, J., Deng, W., Sun, X. et al., 2007. Reaction time of the Continuous Performance Test is an endophenotypic marker for schizophrenia: a study of first-episode neurolepticnaive schizophrenia, their non-psychotic first-degree relatives and healthy population controls.

Schizophrenia research 89 (1), 293-298.

Weigard, A., Huang-Pollock, C. 2014. A diffusion modelling approach to understanding contextual cueing effects in children with ADHD. J Child Psychol Psychiatry 55(12), 1336-1344.

White, C.N., Ratcliff, R., Vasey, M.W., McKoon, G. 2010. Anxiety Enhances Threat Processing Without Competition Among Multiple Inputs: A Diffusion Model Analysis. Emotion, 10(5), 662-677. 


\section{Figure Legends}

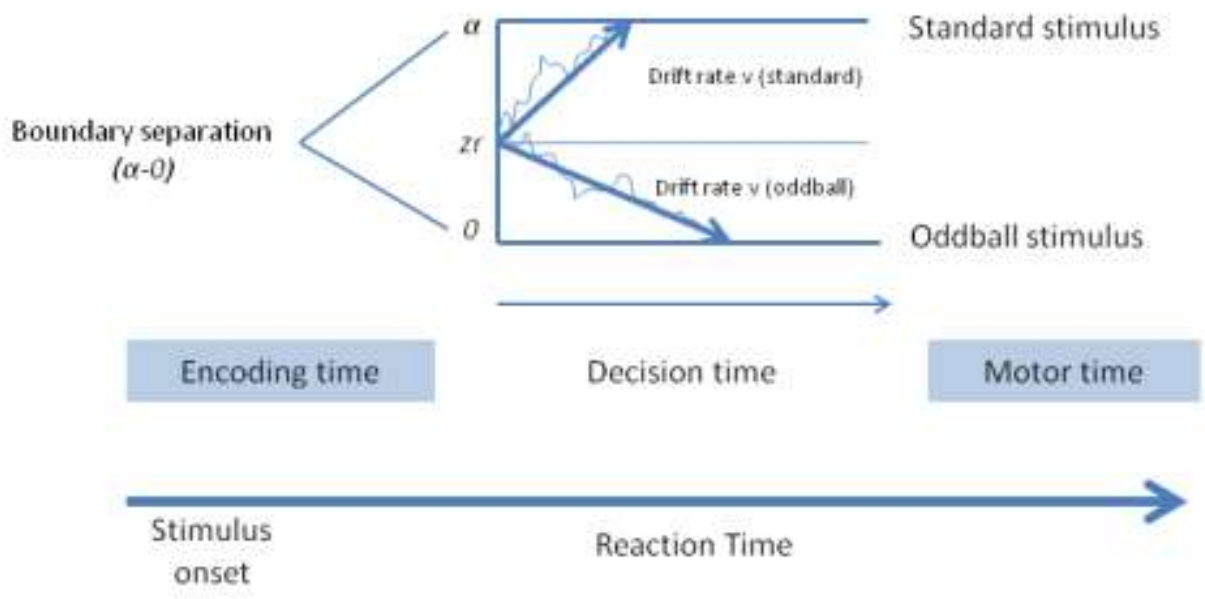

Figure 1. The Drift Diffusion Model: Graphic representation of the application of the Drift Diffusion Model used in this study. The reaction time is modeled as the sum of a decision process and non-decisional processes (stimulus encoding and motor response preparation and execution). The decision process begins at point $z r$ and the rate at which the information is accumulated towards one or the other choices (standard and odd stimulus in this experiment) is the drift rate $v$ (standard) or $v(o d d)$. There are two boundaries for the alternative responses and their separation $(a)$ represents the average amount of required information in order for the participant to reach a decision. The starting point of the decision process is modelled as $z r$. If this point lies in the middle of the two boundaries then there is no bias favouring one of the responses. Finally the combined time for the stimulus encoding and motor response preparation and execution processes is modelled as $t 0$ and its standard deviation is modelled as $s t 0$. 


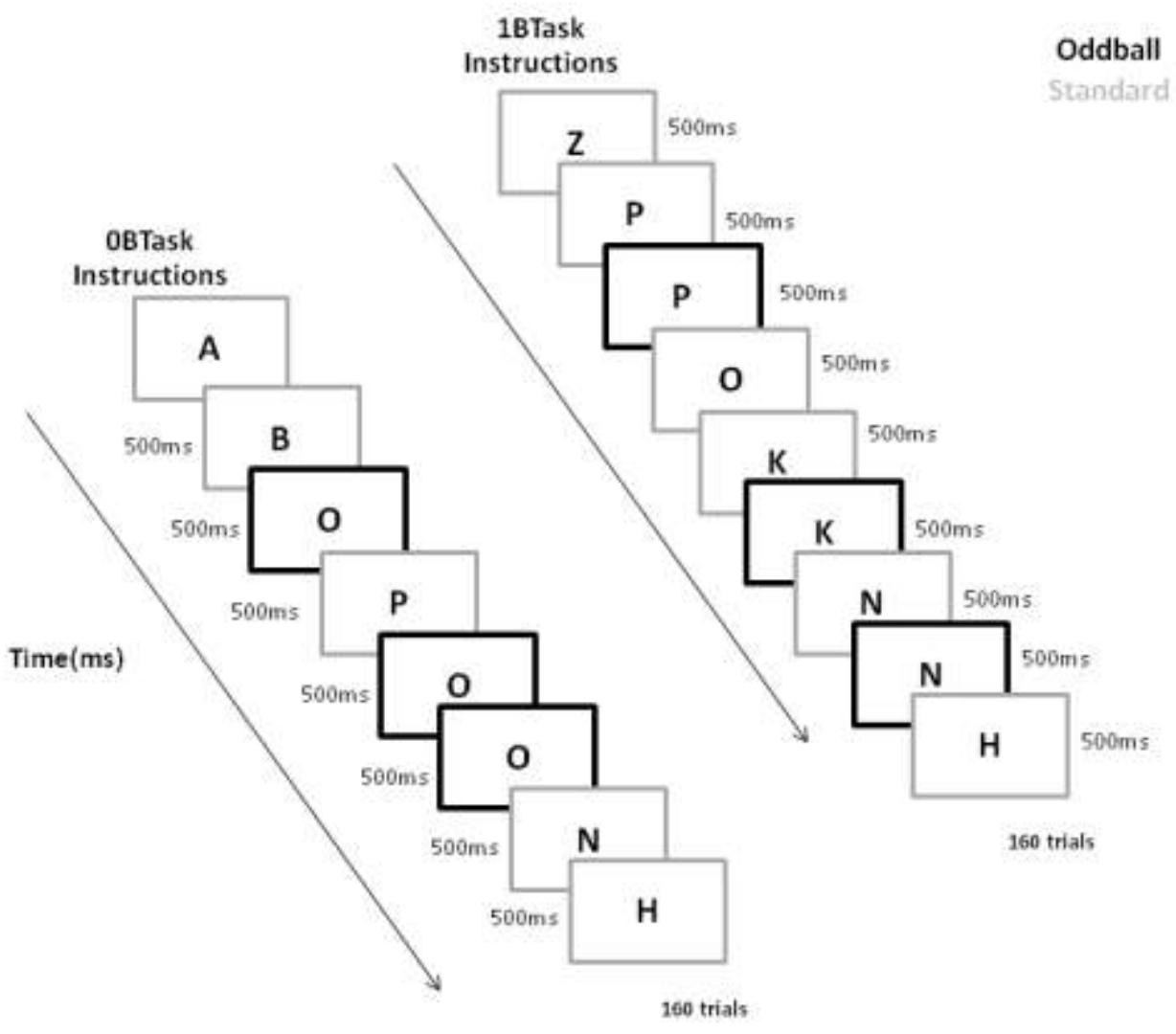

Figure 2. The n-back task: Graphic representation of stimulus sequences in the 0-back and n-back tasks. Oddball trials are marked with black frames and standard trials are marked framed in grey frames. 

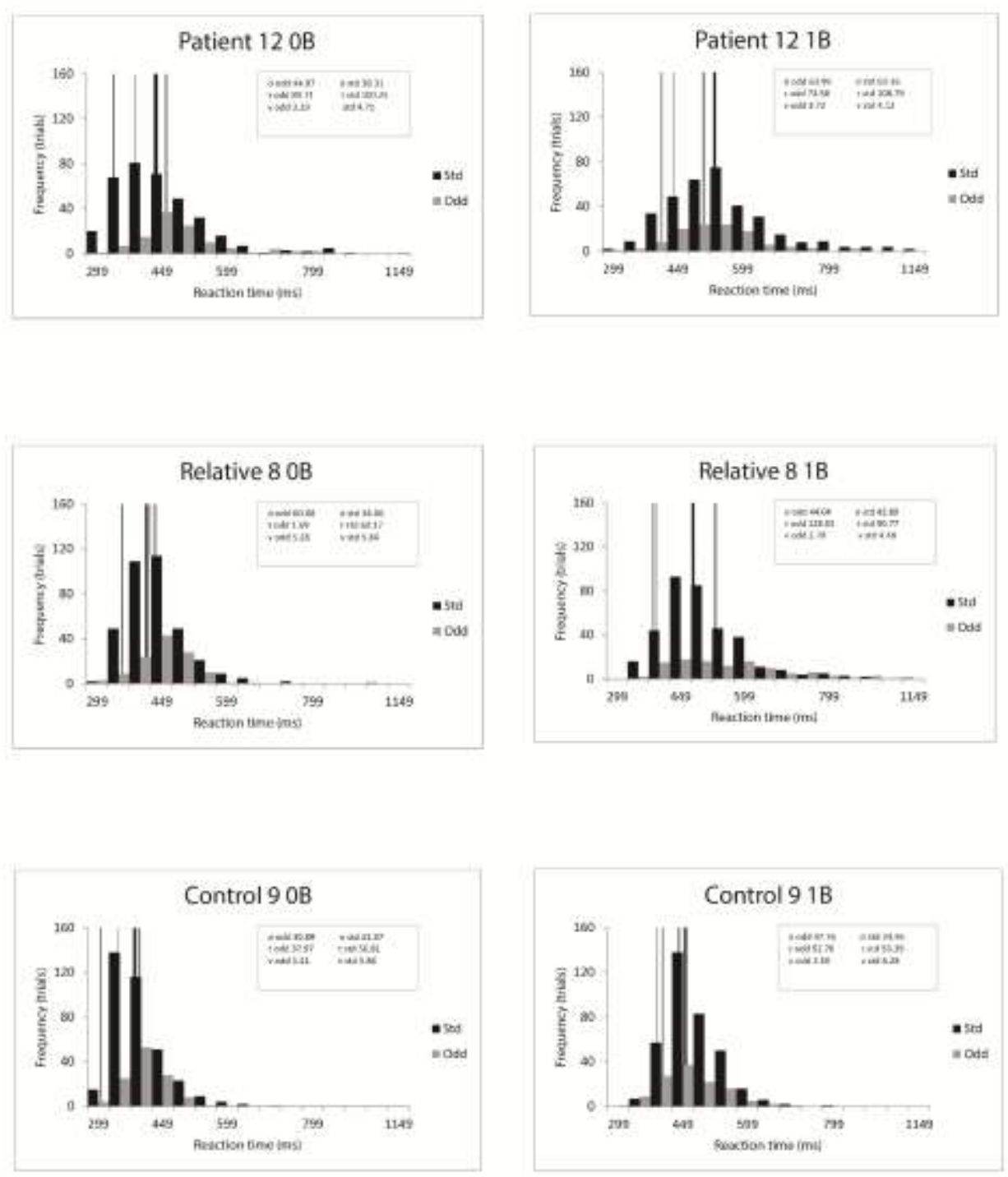

Figure 3. Individual RT-distributions: Histograms displaying the reaction time distribution for one participant from each group for each task. Thick vertical lines displayed on each plot mark the mean reaction time for each trial type (black for standard and gray for oddball) and thin vertical lines mark the $\mathrm{Mu}(\mu)$ also for each trial type. Text boxes in each plot display exGaussian and diffusion model parameters for the same distributions. 
Table 1: Means (SE) for each stimulus condition, task and group for all parameters

\begin{tabular}{|c|c|c|c|c|c|c|c|}
\hline \multirow[t]{2}{*}{ Parameter } & \multicolumn{2}{|c|}{ Stimulus Condition } & \multicolumn{2}{|l|}{ Task } & \multicolumn{3}{|l|}{ Group } \\
\hline & Oddball & Standard & 0-back & 1-back & Patients & Relatives & Controls \\
\hline Accuracy & $93.5(0.6)$ & $99.0(0.2)$ & $98.1(0.2)$ & $94.4(0.6)$ & $94.6(0.6)$ & $97.3(0.7)$ & $96.9(0.5)$ \\
\hline \multicolumn{8}{|c|}{ Gaussian parameters } \\
\hline Mean RT & $459(7)$ & $429(8)$ & $409(8)$ & $479(8)$ & $472(13)$ & $459(15)$ & $401(12)$ \\
\hline RT-SD & $96(3)$ & $106(4)$ & $88(4)$ & $114(3)$ & $112(5)$ & $107(6)$ & \\
\hline \multicolumn{8}{|c|}{ Ex-Gaussian model parameters } \\
\hline $\boldsymbol{\mu}$ & $382(6)$ & $337(6)$ & $340(6)$ & $379(6)$ & 377 (10) & 10 & $329(9)$ \\
\hline $\boldsymbol{\sigma}$ & $49(2)$ & $39(2)$ & $41(2)$ & $47(2)$ & $48(3)$ & & $37(2)$ \\
\hline$\tau$ & $79(3)$ & $92(3)$ & $69(4)$ & $102(4)$ & $95(5)$ & & $72(5)$ \\
\hline \multicolumn{8}{|c|}{ Diffusion Drift Model parameters } \\
\hline$z r$ & - & - & $0.54(.01)$ & $0.46(.01)$ & & $0.49(.02)$ & $0.50(.02)$ \\
\hline$a$ & - & - & $1.19(.04)$ & $1.20(.03)$ & $1.24(.05)$ & $1.20(.05)$ & $1.14(.04)$ \\
\hline$v$ & $3.7(.12)$ & $4.9(.13)$ & $3.7(.11)$ & $4.9(.14)$ & $4.0(.19)$ & $4.1(.22)$ & $4.7(.17)$ \\
\hline to & - & - & $270(6)$ & $309(6)$ & $313(10)$ & $284(11)$ & 271(9) \\
\hline sto & - & - & $104(5)$ & $132(6)$ & $144(9)$ & $117(10)$ & $94(8)$ \\
\hline
\end{tabular}

Note: Accuracy is expressed in percentage of correct responses. Mean RT, RT-SD, $\mu, \sigma$ and $\tau$ are expressed in ms. The diffusion model parameters $z r$ (starting point), $a$ (boundary separation) and $v$ (mean drift rate) are expressed in arbitrary units while $t O$ (mean non-decisional RT) and $s t O$ (SD of the non-decisional RT) are expressed in ms. Standard error of each mean in parentheses. 
Table 2: Independent t-contrasts of planned comparisons for each parameter

\begin{tabular}{|c|c|c|}
\hline \multirow[t]{2}{*}{ Parameter } & $\mathbf{P}+\mathbf{R}$ v C & P v R \\
\hline & t-value (p) & t-value (p) \\
\hline Accuracy & $1.43(0.16)$ & $2.02(0.047)$ \\
\hline \multicolumn{3}{|c|}{ Gaussian parameters } \\
\hline RT(mean) & $4.23\left(<10^{-4}\right)$ & $0.85(0.40)$ \\
\hline RT-SD & $3.76\left(<10^{-3}\right)$ & $0.97(0.33)$ \\
\hline \multicolumn{3}{|c|}{ Ex-Gaussian parameters } \\
\hline $\boldsymbol{\mu}$ & $3.89\left(<10^{-3}\right)$ & $0.44(0.66)$ \\
\hline $\boldsymbol{\sigma}$ & $3.21(0.002)$ & $0.20(0.84)$ \\
\hline$\tau$ & $3.26(0.002)$ & $1.19(0.23)$ \\
\hline \multicolumn{3}{|c|}{ Drift Diffusion Model parameters } \\
\hline$a$ & $1.45(0.15)$ & $1.98(0.05)$ \\
\hline$v$ & $2.86(0.006)$ & $0.13(0.90)$ \\
\hline to & $2.44(0.02)$ & $2.20(0.032)$ \\
\hline sto & $3.49(0.001)$ & $2.10(0.040)$ \\
\hline
\end{tabular}

Note: $\mathrm{P}+\mathrm{R}$ v $\mathrm{C}$ : patients and relatives versus controls contrast, $\mathrm{P}$ v R: patients versus relatives contrast. Significant t-values for each contrast are marked in bold. 